DOSSIÊ TEMÁTICO: Educação Inclusiva e Processos de Subjetivação do/ https://doi.org/10.22481/praxisedu.v16i37.5980

\title{
POR QUE O DIAGNÓSTICO BIOMÉDICO DO TDAH TEM SE TORNADO TÃO DOMINANTE?
}

\author{
WHY HAS ADHD BIOMEDICAL DIAGNOSIS BECOME SO DOMINANT? \\ ¿POR QUÉ EL DIAGNÓSTICO BIOMÉDICO DE TDAH SE HA VUELTO TAN \\ DOMINANTE?
}

Michael W. Corrigan

Marshall University/Ohio State University - Estados Unidos da América

Robert Whitaker

Jornalista investigativo - Estados Unidos da América

Fernando Freitas

Fundação Osvaldo Cruz - Brasil

\begin{abstract}
Resumo: TDAH é hoje a condição de saúde mental mais frequentemente diagnosticada entre crianças e adolescentes. A crença comum é que se trata de uma doença mental, e que médicos e psiquiatras contam com sólidas evidências científicas para dar o diagnóstico e fornecer tratamento psicofarmacológico seguro e eficaz. Porém, a literatura científica não dá suporte a essa crença tão fortemente difundida em nossa sociedade. O artigo faz uma revisão histórica de como essa crença vem sendo construída e analisa um artigo científico que supostamente dá sustentação a esse modo de abordar os problemas comportamentais e emocionais das crianças dos nossos tempos. A conclusão é que a sociedade não conta hoje com evidências que deem suporte a essa crença.
\end{abstract}

Palavras chave: TDAH; modelo biomédico; medicalização.

Abstract: ADHD is today the most frequently diagnosed mental health condition among children and adolescents. The common belief is that it is a mental illness, and doctors and psychiatrists have solid scientific evidence to make the diagnosis and provide safe and effective psychopharmacological treatment. However, the scientific literature does not support this belief so widespread in our society. The article reviews the historical way in which this belief has been constructed and analyzes a scientific article that supposedly supports this way of approaching the behavioral and emotional problems of children of our times. The conclusion is that society today has no evidence to support this belief.

Keywords: ADHD; biomedical model; medicalization. 
Resumen: El TDAH es hoy la afección de salud mental más frecuentemente diagnosticada entre niños y adolescentes. El sentido común es que es una enfermedad mental, y los médicos y psiquiatras tienen evidencia científica sólida para hacer el diagnóstico y proporcionar un tratamiento psicofarmacológico seguro y efectivo. Sin embargo, la literatura científica no respalda esta creencia tan extendida en nuestra sociedad. El artículo revisa la forma histórica en que se ha construido esta creencia y analiza un artículo científico que supuestamente respalda esta forma de abordar los problemas conductuales y emocionales de los niños de nuestro tiempo. La conclusión es que la sociedad actual no tiene evidencia para apoyar esta creencia.

Palabras clave: TDAH modelo biomédico; medicalización.

\section{Introdução}

Algo impactante vem ocorrendo às crianças nas sociedades ocidentais nas últimas décadas. O transtorno do déficit de atenção e hiperatividade (TDAH) se tornou a condição de saúde mental mais frequentemente diagnosticada entre as crianças em idade escolar, atingindo proporções epidêmicas. O diagnóstico é geralmente dado por um psiquiatra infantil ou pediatra que defendem que crianças que apresentam o que consideram ser hiperatividade, desatenção e impulsividade estejam sofrendo de uma condição médica que necessita tratamento com medicação, sobretudo na forma de estimulantes como a Ritalina@, cujas propriedades químicas são similares a várias drogas psicotrópicas de rua. Há drogas mais modernas sendo usadas hoje em dia... Aderall @ é uma das mais populares nestes tempos.

Sem haver algum teste médico para estabelecer quem tem um problema físico (biológico) que esteja causando esses problemas comportamentais, a definição da linha de corte entre normal e TDAH é feita por uma decisão que toma como base o conjunto de sintomas subjetivos que se encontram enumerados nos Manuais oficiais de diagnóstico (DSM Diagnostic and Statistical Manual of Mental Disorders e CID - Classificação Internacional de Doenças). Essas listas de comportamentos usados para definir TDAH aparecem em questionários dados a pais e professores, levando a que, frente aos comportamentos descritos, uma criança ou adolescente seja com muita frequência enquadrado como portador de TDAH (BARKLEY, 2002; SECRETARIA MUNICIPAL DE EDUCAÇÃO DE LUZIÂNIA, 2013; SILVA, 2009). Na verdade, o que esses questionários avaliam é a percepção de um adulto com relação a filho(a), aluno(a), paciente, a uma criança em um momento determinando e em um ambiente específico (CAREY, 1999; BRUSCHMÜLLER et al., 2012; BARKLEY, 2010).

Essa inflação de diagnósticos de TDAH é um fenômeno universal. Segundo A.D.D. Resource Center (2019), nos E.U.A. 6.4 milhões de crianças e adolescentes, entre 4 e 17 anos, foram diagnosticadas com TDAH. A média de idade do diagnóstico de TDAH é de 7 anos de 
idade. A idade quando os sintomas de TDAH tipicamente aparecem é entre 3 e 6 anos. Cerca de $6.1 \%$ das crianças e adolescentes são tratadas com medicamento para TDAH. E nos últimos 8 anos houve um aumento de $42 \%$ de diagnósticos de TDAH.

No Brasil, por sua vez, não há notificações tão precisas quanto nos E.U.A. Não obstante, há estudos proporcionando dados sobre a frequência, a severidade e o curso de tratamento em relação ao gênero, idade, área geográfica, fatores psicossociais, etc. (DIAS, JIMÉNEZ, RODRÍGUEZ, \& AFONSO, 2013; FARAONE, SERGEANT, GILBERG, \& BIEDERMAN, 2003; HODGKINS et al., 2012). Estima-se que 4.4\% das crianças e adolescentes brasileiras, entre 4 e 18 anos, têm o diagnóstico de TDAH, segundo estudo coordenado pelo Instituto Gila feito com 5.961 crianças e adolescentes em 18 estados do país (ESTADÃO, 2011).

Neste artigo tomaremos como foco a narrativa dominante que o TDAH é uma condição médica cuja causalidade vem sendo identificada. Consideramos que valha a pena fazer uma breve reconstrução histórica de como os comportamentos de hiperatividade, desatenção e impulsividade foram sendo objeto da atenção médica. O senso-comum é que se trata de uma síndrome heterogênea de origem multifatorial, integrando fatores genéticos, neurobiológicos, ambientais e múltiplos genes associados. Há um fator que é da maior relevância: mesmo com os avanços dos estudos genéticos e das técnicas de neuroimagem, não há consenso sobre a etiologia biológica do TDAH. Busca-se dramaticamente por biomarcadores para o TDAH. E ainda que não existam evidências seguras e confiáveis de uma determinação biológica, o que chega ao grande público é que há tais evidências. Um exemplo eloquente é o estudo publicado por The Lancet Psychiatry, em 2017, que teve uma forte repercussão na grande mídia. Segundo o que foi divulgado para o público, finalmente havia sido descoberto diferenças significativas nos cérebros entre os diagnosticados com TDAH e os 'normais'. Iremos aqui reapresentar a análise deste artigo que já fizemos em outra ocasião e assim permitir que o público de língua portuguesa seja melhor informado sobre o que a Ciência mostra. E, finalmente, iremos fazer algumas considerações finais.

\section{Uma breve reconstrução do diagnóstico de TDAH}

Hiperatividade, desatenção e impulsividade em crianças foram primeiramente conceitualizadas como sendo fenômeno médico nos anos iniciais do século passado (STIL1, 1902). A hiperatividade e a desatenção em crianças são comportamentos que chegaram a ser até mesmo vistos como relacionados ao ‘diagnóstico de dano cerebral mínimo’ (MBD), sendo a medicação psíquico-estimulante o tratamento recomendado. Nos anos 1960, a segunda edição 
do DSM apresentará a categoria diagnóstica 'reação hipercinética infantil' para substituir o diagnóstico MDB (AMERICAN PSYCHIATRIC ASSOCIATION, 1966). No começo dos anos 1980, com o DSM-III, surge uma renomeação, desta vez designada como 'transtorno de déficit de atenção' (TDA), que poderia ser diagnosticado com ou sem hiperatividade. O diagnóstico deveria ser definido usando três dimensões (três separadas listas de sintomas), uma para déficits de atenção, uma para impulsividade e uma para hiperatividade (DSM-III, AMERICAN PSYCHIATRIC ASSOCIATION, 1980). Essa abordagem tridimensional foi abandonada, ao final dos anos 1980, quando o DSM-III foi revisto e se tornou DSM-III-R (AMERICAN PSYCHIATRIC ASSOCIATION, 1987), em favor de uma combinação de sintomas em apenas uma lista (uma dimensão). Surge o termo 'transtorno de déficit de atenção e hiperatividade (TDAH), com atenção, hiperatividade e impulsividade a partir de então sendo assumidos como parte de um único transtorno sem distinções. Quando apareceu a $4^{\mathrm{a}}$. ediç̧ão do DSM (DSM-IV, AMERICAN PSYCHIATRIC ASSOCIATION, 1994), os critérios de diagnóstico do TDAH serão uma vez mais reconsiderados, desta vez em favor de um modelo bidimensional com o déficit de atenção sendo uma subcategoria e hiperatividade-impulsividade a outra.

Atualmente, a definição técnica formal para TDAH é aquela encontrada na $5^{\mathrm{a}}$. edição do DSM (AMERICAN PSYCHIATRIC ASSOCIATION, 2013). Os núcleos dos domínios sintomáticos são os mesmos do DSM-IV (déficit de atenção e hiperatividade/impulsividade), bem como os 18 sintomas principais (CORRIGAN, 2015). Porém, inclui modificações para cada um dos critérios de diagnóstico (A-E) do TDAH, ampliando ainda mais o escopo da abrangência das pessoas virtualmente diagnosticáveis com TDAH. O Critério A (sintomas do TDAH) é mantido, exceto pela inclusão de mais exemplos de comportamentos que podem se manifestar na adolescência e na vida adulta e pela redução de 6 para 5 no número mínimo de sintomas, em cada um dos domínios de sintomas requeridos, para o adolescente mais velho e adultos a partir de 17 anos. O critério $B$ (idade de início) mudou de "início de sintomas e comprometimentos antes dos 7 anos" para "início de sintomas antes dos 12 anos". O critério $C$ (abrangência) foi alterado de "evidência de comprometimento" para "evidência de sintomas em dois ou mais contextos". O critério $D$ (comprometimento) agora exige que os comprometimentos funcionais precisem "reduzir a qualidade do funcionamento social, acadêmico ou ocupacional" em vez de exigir que sejam "clinicamente significativos". O critério $E$ (condições de exclusão) não inclui mais o Transtorno do Espectro do Autismo como um diagnóstico excludente. Com relação à nosologia, os 'tipos' de DSM-IV TDAH agora são 
chamados de 'apresentações'. Finalmente, foram adicionados modificadores para que a gravidade do transtorno (por exemplo, leve, moderada ou grave) possa ser especificada e o transtorno possa ser codificado como 'em remissão parcial' se os critérios diagnósticos completos não forem atendidos no momento (EPSTEIN \& LOREN, 2013).

\section{O TDAH tem uma causa biológica definida?}

Uma barreira enorme para se aceitar TDAH como uma doença é que sua causa é desconhecida, não havendo um biomarcador. Uma variedade de teorias tem aparecido para dar conta dos comportamentos de desatenção, impulsividade e hiperatividade, que são considerados como sintomas do TDAH. Que incluem: (1) abordar a desatenção enquanto um transtorno da aprendizagem devido a deficiências no 'desenvolvimento neurológico'; ou (2) que a sintomatologia que caracteriza o TDAH é expressão de traços da personalidade; ou (3) uma variação no comportamento normal; ou (4) evidências de taxas distintas de amadurecimento do desenvolvimento cerebral; ou (5) resultantes de expectativas mais ou menos estereotipadas dos pais e dos educadores nas sociedades dos tempos contemporâneos.

Contudo, é a busca por uma causalidade biológica o que mais tem recebido incentivo para a pesquisa. É a visão medicalizante que domina o imaginário social da sociedade contemporânea. Por que esse modo particular de ver as crianças/adolescentes e de lidar com os seus problemas tem se tornado tão dominante?

O estudo que analisaremos a seguir é eloquente. Os resultados divulgados concluem que indivíduos com TDAH têm cérebros menores (LANCET PSYCHIATRY, 2017). Esse estudo foi propagandeado como um grande avanço científico.

\section{O estudo publicado pela Lancet Psychiatry}

Este foi um grande estudo internacional, financiado pelos Institutos Nacionais de Saúde. Martine Hoogman e seus 81 coautores realizaram uma análise de dados secundários das ressonâncias magnéticas usadas para medir os volumes cerebrais em 1713 pacientes diagnosticados com TDAH e em 1529 indivíduos que não tiveram esse diagnóstico. Esses dados foram coletados em 23 sites no mundo inteiro. Os autores escreveram que seu estudo, "usando o maior conjunto de dados até o momento", documentou que "os volumes do accumbens, amígdala, caudado, hipocampo, putâmen e volume intracraniano eram menores em indivíduos 
com TDAH em comparação com os indivíduos do grupo de controle" (Hoogman et al., 2017, p. 1).

Esses resultados, concluíram os autores, continham mensagens importantes para os médicos:

Os dados da nossa análise muito bem fundamentada confirmam que os pacientes com TDAH apresentam cérebros alterados e, portanto, que o TDAH é um distúrbio do cérebro. Esta mensagem é clara para os médicos transmitirem aos pais e pacientes, o que pode ajudar a reduzir o estigma de que o TDAH é apenas um rótulo para crianças difíceis e causado por uma paternidade incompetente. Esperamos que este trabalho contribua para uma melhor compreensão do TDAH no público em geral. (HOOGMAN et al., 2017, p. 7).

Ao longo do artigo existem muitas afirmações sugerindo que este estudo fornece evidências de que volumes cerebrais menores são específicos para indivíduos com diagnóstico de TDAH. Em sua análise, os autores também declararam que haviam investigado a exposição da coorte de TDAH a medicamentos estimulantes e verificaram que os medicamentos não desempenhavam nenhum papel como uma possível causa dos volumes menores. "Refutamos os efeitos da medicação no volume cerebral sugeridos por metanálises anteriores”, escreveram (Hoogman et al., 2017, p. 1).

Os comunicados de imprensa enviados à mídia refletiram as conclusões do artigo, e as manchetes relatadas pela mídia, por sua vez, resumiram com precisão os comunicados de imprensa.

\section{Comunicados de Imprensa}

\begin{tabular}{|c|c|c|c|}
\hline The Telegraph: & Newsweek: & WebMD: & $C N N$ : \\
\hline $\begin{array}{l}\text { “TDAH é } \\
\text { um transtorno } \\
\text { cerebral, não um } \\
\text { rótulo para uma } \\
\text { educação } \\
\text { deficiente, dizem } \\
\text { cientistas” }\end{array}$ & \begin{tabular}{|l} 
“Estudo descobre \\
que os cérebros \\
de pessoas que \\
sofrem com TDAH \\
são menores”
\end{tabular} & $\begin{array}{l}\text { “O estudo de imagem } \\
\text { confirma as } \\
\text { diferenças cerebrais } \\
\text { em pessoas com } \\
\text { TDAH” }\end{array}$ & \begin{tabular}{|l} 
“Os cérebros de \\
pessoas com \\
TDAH apresentam \\
estruturas \\
relacionadas à \\
emoção menores”
\end{tabular} \\
\hline $\begin{array}{l}16 \text { de Fevereiro de } 2017 \\
\text { Por Henry Bodkin }\end{array}$ & \begin{tabular}{|l}
16 de Fevereiro de 2017 \\
Por Conor Gaffey
\end{tabular} & $\begin{array}{l}15 \text { de Fevereiro de } 2017 \\
\text { Por Robert Preidt }\end{array}$ & $\mid \begin{array}{l}15 \text { de Fevereiro de } 2017 \\
\text { Por Susan Scutti }\end{array}$ \\
\hline
\end{tabular}

Fontes: The Telegraph (https://www.telegraph.co.uk/science/2017/0...); $\quad$ Newsweek: https://www.newsweek.com/brains-adhd-sufferers-are-smaller-suggesting-it-physical-disorder-study557372; WebMD: https://www.webmd.com/add-adhd/news/20170215/imaging-study-confirms-braindifferences-in-people-with-adhd; CNN: https://www.cnn.com/2017/02/15/health/adhd-brain-scansstudy/index.html 
Juntas, as reivindicações dos autores e as manchetes da mídia contribuem para uma poderosa mensagem para o público: os pais de crianças diagnosticadas passam a perceber que há algo errado com o cérebro de seus filhos. $\mathrm{E}$ as próprias crianças e adolescentes passam a entender ser essa a verdade, e esse ‘conhecimento' definirá ainda mais o senso de si próprias. Enquanto Hoogman e colegas afirmaram que esse entendimento "reduzirá o estigma do TDAH", o oposto é provavelmente o mais verdadeiro: crianças e adolescentes diagnosticadas com TDAH são anormais, na medida em que sofrem de um 'cérebro menor', inferior.

Mas é fato que o estudo feito apoia de fato a conclusão de que crianças e adolescentes com TDAH têm 'cérebros alterados'? Há nessas crianças volumes menores em diferentes regiões do cérebro delas? Será que autores apresentaram dados que 'refutam' convincentemente estudos anteriores sugerindo que a exposição a medicamentos pode ser uma causa de menores volumes cerebrais?

\section{Análise dos Resultados}

Para começar esta revisão, começaremos com uma descoberta surpreendente escondida em um local incomum - o apêndice do estudo. Podemos então imaginar como seriam as manchetes da mídia se os autores houvessem se concentrado nesses dados. O título alternativo poderia ser: "Grande estudo descobre que crianças com TDAH têm um QI mais alto!".

Porém, para se deparar com essa descoberta você necessita gastar US\$ 31,50 para comprar o artigo e, em seguida, fazer um pedido especial à Lancet Psychiatry para que o apêndice seja enviado. Em seguida, você descobrirá, nas páginas 7 a 9 do apêndice, uma "Tabela 2" que fornece escores de QI para a coorte de TDAH e os indivíduos do grupo de controle.

Embora o estudo tenha sido feito em 23 locais clínicos, apenas 20 relataram dados comparativos de QI. Em 16 dos 20, a coorte de TDAH teve QI mais alto, em média, do que o grupo controle. Nas outras quatro clínicas, o TDAH e os grupos controle tiveram o mesmo QI médio (com os escores médios de QI dos dois grupos no intervalo de dois pontos um do outro). Assim, em todos os 20 locais, o grupo TDAH apresentou um escore médio de QI que foi igual ou superior à pontuação média de QI para o grupo controle. 


\section{Escores de QI}

\begin{tabular}{|c|c|c|}
\hline \multicolumn{3}{|c|}{ Pontuação de QI } \\
\hline Locais & TDAH coorte & Controle \\
\hline Wurtzburgo, Alemanha & 117 & 114 \\
\hline Dublim, Imanda & 113 & 103 \\
\hline Baltimore, EUA & 112 & 107 \\
\hline Nowa borque, EUA & 111 & 106 \\
\hline Pequim, China & 118 & 106 \\
\hline Öregon, EUA & 116 & 110 \\
\hline Aachen, Alemanha & 110 & 102 \\
\hline Bergen, Noruega & 116 & 111 \\
\hline Bergen, Noruega (2) & 96 & 97 \\
\hline Londires, inglaterra & 114 & 109 \\
\hline Nimega, Holanda & 110 & 107 \\
\hline Nowa lorque, EUA & 113 & 115 \\
\hline Utrecht, EUA & 106 & 102 \\
\hline Nowa lorque, EUA & 112 & 111 \\
\hline Mannheim, Alemanha & 120 & 110 \\
\hline Londires, Inglaberra & 110 & 92 \\
\hline Amstendâ, Holanda & 105 & 95 \\
\hline Nimega, Holanda & 109 & 98 \\
\hline NIH & 108 & 108 \\
\hline MTA & 108 & 103 \\
\hline
\end{tabular}

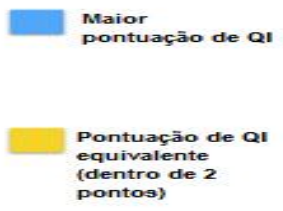

Fonte: Hoogman et al., 2017, Appendix, Table 2

A suposição usual é que as crianças com TDAH, que sofrem de um 'distúrbio cerebral', são menos capazes de se concentrar e de ter um foco em suas atividades na escola e, portanto, que são prejudicadas de alguma forma. Os autores deste estudo relataram descobertas que mostram que o TDAH é um distúrbio do cérebro. Mas se a pontuação média de QI da coorte de TDAH é maior que a pontuação média dos indivíduos dos grupo de controle, essa suposição básica não precisaria ser reavaliada? Se os participantes com TDAH têm cérebros menores, repletos de 'estruturas alteradas', como eles são tão espertos quanto, ou até mais inteligentes, que os participantes do grupo de controle?

Os autores, no entanto, optaram por enterrar os dados de QI em um apêndice, que não é facilmente obtido. Mesmo após a compra do documento, é necessário fazer um pedido especial para obter o apêndice. Por quê? E por que os autores não discutiram os dados de QI em seu artigo ou os utilizaram em suas análises? Quando uma investigação científica leva a um resultado surpreendente que basicamente contradiz a principal alegação do estudo, os autores têm o dever - em termos de adesão aos valores éticos supostamente governados pela ciência de apresentar esses resultados. Mas os autores deste estudo não fizeram isso.

De fato, se os dados de QI tivessem sido promovidos no resumo do estudo e junto à mídia, o público estaria agora tendo uma nova discussão: é possível que crianças diagnosticadas com TDAH sejam mais inteligentes que a média? Eis uma manchete que segundo as evidências desse estudo poderia estar nos principais órgãos da grande mídia:

Talvez estejamos drogando milhões de crianças brilhantes porque elas são mais propensas ao tédio e as escolas não estão proporcionando ambientes estimulantes de aprendizado. 
Uma outra questão bastante relevante deste estudo é com respeito ao tamanho do cérebro em crianças com o diagnóstico de TDAH. Tomando como referência o que foi alardeado pela grande mídia: "Estudo descobre que cérebros de pessoas que sofrem de TDAH são menores", uma revisão dos ‘tamanhos de efeito' relatados revela que os autores do artigo não encontraram tais resultados.

O que se supõe é que os pesquisadores devem ter estabelecido um volume 'normal' (que seria o volume cerebral médio de um grupo controle) e, em seguida, determinaram que a maioria, se não todos, daqueles diagnosticados com TDAH têm volumes cerebrais menores do que a norma. Mas esse não foi o caso aqui.

Neste estudo, os autores reuniram dados de ressonância magnética cerebral dos 3.242 participantes do estudo (que foram coletados e arquivados nos 23 locais) e depois calcularam, para cada coorte, os volumes intracranianos médios e os volumes médios de regiões específicas do cérebro. Eles relataram as diferenças para cada uma dessas comparações e o "tamanho do efeito" das diferenças. Esse é o aspecto crítico dos resultados a serem considerados e compreendidos: os tamanhos dos efeitos revelam a verdadeira força dos resultados e a quantidade de sobreposições entre os volumes cerebrais individuais nos dois grupos e, portanto, estabelecem a probabilidade de um indivíduo no grupo TDAH tenha um volume cerebral menor do que um indivíduo no grupo controle.

Por exemplo, os autores relataram um tamanho de efeito de Cohen de 0,19 para diferenças no volume médio dos núcleos accumbens em crianças menores de 15 anos. Segundo os autores, "os núcleos accumbens, com seu papel proeminente no processamento de recompensas, é central para motivações e disfunção emocional em pacientes com TDAH " (p. 7). Os tamanhos de efeito d de Cohen variam de zero a três e, portanto, 0.19 é considerado um pequeno efeito. No entanto, neste estudo, para jovens com menos de 15 anos, foi o maior tamanho de efeito de todas as comparações de volume cerebral que foram feitas. Especificamente, sobre o significado desse tamanho de efeito de 0. 19:

- Aproximadamente 58\% dos jovens com TDAH nessa amostra de conveniência tinham um volume accumbens abaixo da média no grupo controle, enquanto $42 \%$ dos jovens com TDAH tinham um volume accumbens acima da média no grupo controle.

- $\quad$ Além disso, se você soubesse o volume accumbens de uma criança escolhida aleatoriamente, teria $54 \%$ de chance de adivinhar corretamente a qual das duas coortes - TDAH ou controle saudável - a criança pertencia. 
Em resumo, se você jogasse uma moeda para adivinhar a qual das duas coortes a criança pertencia, suas chances de acertar seriam quase as mesmas. O valor diagnóstico de uma ressonância magnética cerebral, com base nos achados deste estudo, teria tanto pouco valor preditivo quanto o lançamento de uma moeda.

Existem maneiras de visualizar a sobreposição desses dados. Se você traçasse as medidas individuais de accumbens para todas as 1.637 crianças menores de 15 anos neste estudo e usasse um ponto vermelho para marcar os participantes do TDAH e um ponto preto para marcar os de controle, você veria uma confusão de pontos vermelhos e pretos. Haveria uma porcentagem ligeiramente mais alta de pontos vermelhos localizada na metade inferior da escala e uma porcentagem ligeiramente mais alta de pontos pretos na metade superior, mas você poderia ver imediatamente - pela confusão de pontos - que 'pequeno volume cerebral' não era uma característica distintiva dos indivíduos da coorte de TDAH. Os volumes cerebrais individuais variaram bastante, e isso é verdadeiro para as duas coortes, e todos os dados reunidos mostraram que houve uma chance um pouco maior para qualquer criança individual diagnosticada com TDAH, em comparação com uma criança na coorte de controle, houvesse um medida do accubens sido traçada na metade do volume mais baixo do gráfico.

\section{Tamanho do Efeito Cohen}

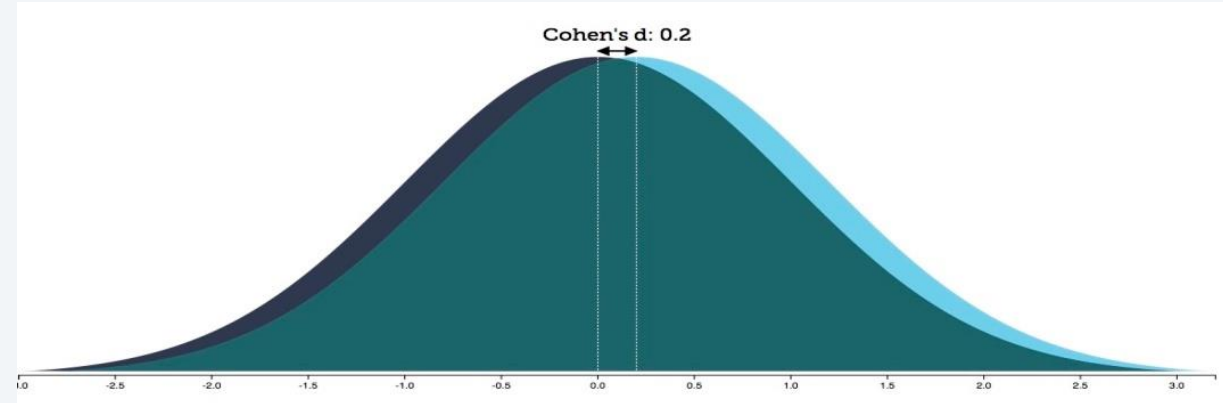

Fonte: Graphic by Kristoffer Magnusson, http://rpsychologist.com/d3/cohend/

Os tamanhos dos efeitos para as outras sete comparações de volume cerebral feitas em crianças menores de 15 anos variaram de 0,01 a 0,18 . Todos os tamanhos do efeito foram bem pequenos. E com essa compreensão do que os tamanhos do efeito revelam, é fácil ver que as descobertas, apresentadas no estudo e na mídia, são completamente enganosas. Os autores fizeram parecer que uma criança diagnosticada com TDAH tem um volume cerebral menor, é claro, e que essa é a característica definidora do transtorno, quando, de fato, os autores descobriram que os volumes cerebrais individuais dos dois grupos se sobrepuseram amplamente. 
Com o tamanho de efeito d de Cohen de 0,1 , como foi o caso das comparações de volume cerebral de paládio em crianças de 15 anos ou menos, haveria uma sobreposição de $96 \%$ entre os dois grupos.

\section{Tamanho do Efeito Cohen}

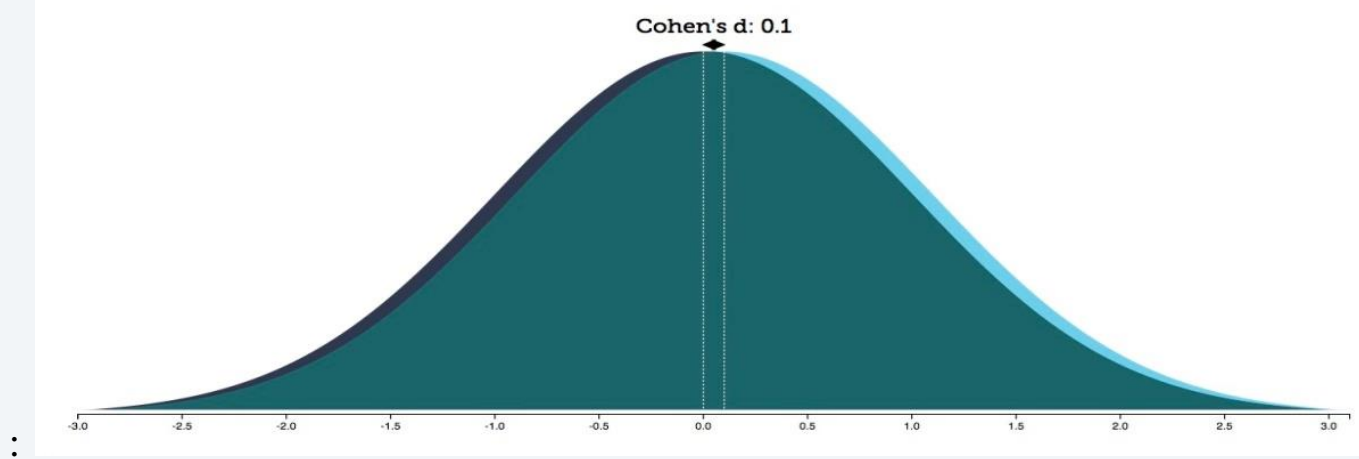

Fonte: Graphic by Kristoffer Magnusson, http://rpsychologist.com/d3/cohend/

Agora, voltemos à afirmação feita por Hoogman e colegas de que seu estudo mostrou que os volumes cerebrais são "menores em indivíduos com TDAH em comparação com os de grupo de controle". O tamanho do efeito d de Cohen de tal descoberta seria 3, porque é uma afirmação que implica que todos aqueles com TDAH têm volumes menores que o volume médio dos do grupo de controle. Nesse caso, haveria apenas uma sobreposição de $13 \%$ entre as curvas de distribuição dos dois grupos.

\section{Tamanho do Efeito Cohen}

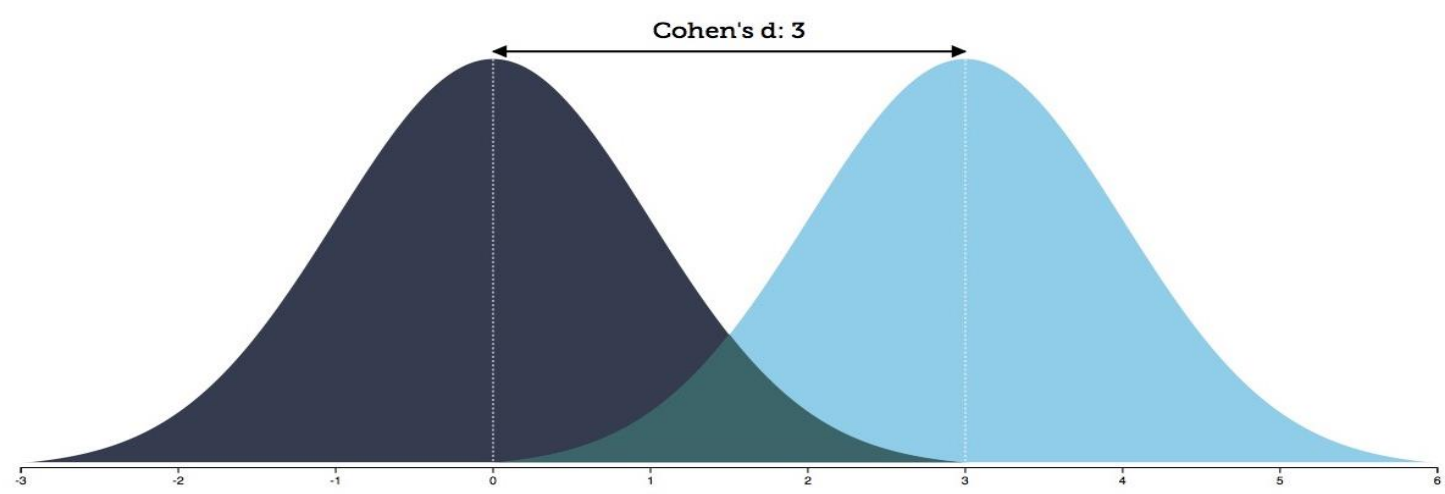

Fonte: Graphic by Kristoffer Magnusson, http://rpsychologist.com/d3/cohend/

A falácia científica do estudo é a seguinte: você não pode pegar dados que indiquem uma diferença média em volumes cerebrais agrupados com tamanho de efeito pequeno e 
generalizar essa diferença para indivíduos da coorte de TDAH e apresentá-los - como Hoogman e colaboradores fizeram (Hoogman et al., 2017) como uma característica definidora do TDAH.

\section{O efeito da medicação}

Como observado acima, as descobertas dos autores mostram que houve pequenas diferenças nos volumes cerebrais médios de crianças com TDAH e no grupo controle. Estudos anteriores sugeriram que os medicamentos para o TDAH poderiam reduzir os volumes cerebrais e, portanto, Hoogman e colaboradores avaliaram se as pequenas diferenças nos volumes cerebrais médios poderiam ser devidas à exposição a esses psicoestimulantes.

Para fazer isso, eles compararam os volumes cerebrais médios de dois grupos na coorte de TDAH: 82 que disseram que nunca usaram medicamentos estimulantes (ingênuos) e 637 que disseram que "usavam medicamentos estimulantes em algum lugar da vida durante um período de mais de quatro semanas" (exposto ao medicamento). Os autores relataram que "não houve diferenças em nenhum dos volumes" entre os grupos ingênuos e expostos a medicamentos e, portanto, concluíram que o estudo "refutou" os estudos anteriores. (Hoogman et al., 2017, p.5).

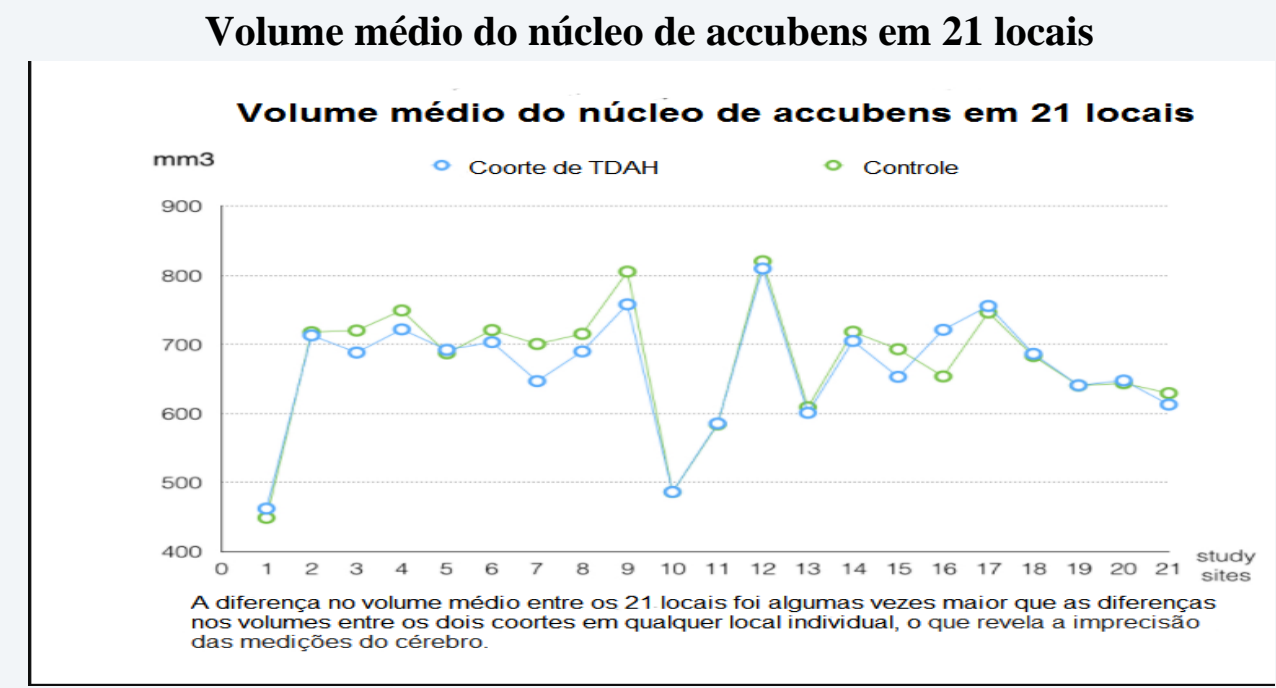

Fonte: Hoogman et al (2017).

Mas há deficiências notáveis na execução e relato dessa análise. Especificamente: eles não publicaram os dados médios do volume para os dois grupos. Eles simplesmente declararam que os volumes eram os mesmos.

Eles não informaram quantos pacientes expostos a medicamentos eram crianças $\mathrm{e}$ quantos eram adultos. Dado que foi principalmente em crianças menores de 15 anos que houve 
diferenças "estatisticamente significativas" nos volumes cerebrais médios entre o TDAH e os controles, seu esforço para verificar se a exposição à medicação era um fator nessas diferenças deveria ter uso isolado de medicação nessa faixa etária. .

Eles não forneceram nenhuma informação relacionada à dosagem para o grupo exposto à medicação ou informações sobre quanto tempo eles tomaram os medicamentos. Se uma pessoa de 30 anos tivesse tomado um estimulante por quatro semanas em criança, seria de esperar que isso realmente tivesse um efeito a longo prazo no volume cerebral? E mais ao ponto: havia diferenças de volume entre as crianças com "TDAH" que usavam drogas há vários anos e as crianças da coorte de TDAH que nunca as usavam? Esse é o tipo de comparação que precisava ser feito.

Há um grupo ausente de pacientes nessa comparação. Em um ponto do artigo, os autores afirmaram que possuíam informações sobre o uso de medicamentos para 1254 dos 1713 participantes do grupo TDAH. No entanto, sua comparação envolveu apenas 719 pacientes (82 mais 637). Por que eles excluíram 545 pacientes (1254 menos 719) dessa comparação?

Sem dados mais abrangentes apresentados e perguntas relacionadas a dados ausentes respondidas nas seções de métodos ou resultados, não há como avaliar se este estudo fornece uma "refutação" das preocupações de que os medicamentos para o TDAH possam diminuir o volume cerebral ao longo do tempo. Apenas nos pedimos que tomemos a palavra dos autores e isso não é um exemplo de como você valida uma descoberta científica para uma questão controversa.

Poderíamos aqui apresentar uma análise crítica que fizemos dos dados individuais que escondem a conclusão declarada pelos autores. Para quem tem interesse em aprofundar a análise detalhada que fizemos dos resultados apresentados pelos autores com exames de ressonância magnética, recomendamos que acessem o que publicamos no site do madinamerica (Corrigan \& Whitaker, 2017).

Resumindo. Para este estudo, é explicado e entendido que existe um grupo que tem TDAH e um grupo de controle que não. Mas, como não há marcador biológico que possa ser usado para fazer esse diagnóstico, como foi feita essa distinção?

A seção de métodos no artigo publicado não fornece nenhuma informação sobre esta questão crítica. Em vez disso, os autores simplesmente escrevem que "procedimentos de diagnóstico para cada site estão listados no apêndice” (Hoogman et al, 2017, p. 3).

Portanto, que você volte novamente para a Tabela 2 no apêndice e o que você irá constatar é que não houve nenhum método de diagnóstico padronizado aplicado em todos os 
locais. Em vez disso, essa distinção crítica - TDAH versus nenhum TDAH - foi feita de maneira aleatória.

Primeiro, dois dos 23 sites nem sequer tinham um grupo de controle. Portanto, é difícil entender por que as medidas de TDAH desses dois sites foram incluídas nos dados agrupados.

Segundo, parece que nenhum dos participantes dos grupos de controle nos 21 locais restantes recebeu uma avaliação diagnóstica para o TDAH. Não há relatos de pontuações de sintomas de TDAH para os controles. Os participantes rotulados como "controles saudáveis" e, portanto, vistos como não tendo TDAH - aparentemente nunca foram testados para verificar se exibiam os comportamentos associados a esse diagnóstico.

Terceiro, os autores não testaram quase mil dos participantes da coorte de controle para determinar se eram "saudáveis". Eles listaram 867 na coorte de controle como desconhecida, relacionada a problemas de comorbidade, como depressão, ansiedade e abuso de substâncias. Sem esses testes, não parece que esse grupo "sem TDAH" possa ser descrito como "controles saudáveis". De fato, há informações muito limitadas sobre os controles. Por que esses indivíduos concordaram em participar deste estudo? Eles foram recrutados por meio de anúncios que prometiam pagamento? Ou eles eram pacientes nas clínicas que estavam recebendo uma ressonância magnética por outros motivos médicos? No apêndice, os autores afirmaram que 30 controles foram diagnosticados com depressão, $11 \mathrm{com}$ ansiedade e 39 com transtornos por uso de substâncias. Porém, com tão poucas informações abrangentes fornecidas, é impossível saber quão representativo dos "controles saudáveis" esse grupo é.

Quarto, em sete dos 23 sites, não existem pontuações de sintomas de TDAH listadas na coorte de TDAH. Só se pode adivinhar como foi feito o diagnóstico nesses locais. Os autores possuíam registros dos médicos dos participantes? Ou eles confiaram no autodiagnóstico ou no relato dos participantes de que tinham TDAH? Não há como saber.

Quinto, mesmo quando os escores de gravidade dos sintomas foram relatados, não havia padronização do "instrumento" usado para avaliar os sintomas ou do sistema de classificação usado para fazer o diagnóstico (DSM IV ou CID 10). Em outras palavras, os autores de uma clínica no Brasil podem ter um padrão para o diagnóstico de TDAH, e os autores na China, um segundo padrão, e os autores no Reino Unido, um terceiro, e assim por diante.

\section{Considerações finais}

O nosso entendimento dos comportamentos chamados de hiperatividade, déficit de atenção e impulsividade, que com uma relativa frequência aparecem na infância e na 
adolescência, é moldado pelas crenças de uma sociedade. Essas crenças culturais são influenciadas pelos nossos sistemas de valores que influenciam o sentido que damos a um problema e o que fazemos para lidar com ele. A sociedade contemporânea está fortemente dominada pelo imaginário da 'medicalização' (Freitas e Paulo Amarante, 2017). Quer dizer, cada vez mais é 'senso-comum' se efetuar a passagem de uma condição social ou um comportamento individual - considerados como indesejáveis ou perturbadores - ao estatuto de patológico. E isso é feito sobre bases supostamente científicas. Porém, o que a Ciência sugere é que esse processo de 'medicalização' é na verdade um sintoma de uma cultura 'disfuncional', ao invés de ser um progresso científico devido à descoberta de transtornos médicos e formas de como trata-los. O diagnóstico TDAH tem pouca evidência científica para dar suporte à ideia que ele representa um transtorno físico do cérebro e do sistema nervoso. A análise crítica das evidências científicas hoje existentes concluem que esse diagnóstico nos diz muito pouco (se não nada) a respeito da causa, do tratamento e resultados para tais problemas emocionais ou comportamentais. A análise crítica que fizemos do artigo publicado por Lancet Psychiatry é ilustrativa.

O sistema de classificação que hoje é utilizado para categorizar o TDAH na verdade não se desenvolveu como um resultado de nossas descobertas, mas sim de uma mudança no modo como nós pensamos e categorizamos as emoções e comportamentos das crianças e adolescentes. Quer dizer, é resultante de uma nova 'construção social' da infância. Ou, com outras palavras: da 'medicalização' da vida.

Tem sido em vão a busca por evidências biológicas para dar suporte a essa nova forma de lidar esse modo de como estamos lidando com a infância e a adolescência. Não obstante esse espetacular fracasso para descobrir evidências científicas confiáveis e replicáveis que o TDAH é resultado de anormalidades genéticas, bioquímicas, desenvolvimentais, do cérebro, isso não tem impedido o crescimento da crença que o TDAH seja, no entanto, o resultado de anormalidades conhecidas no cérebro.

Vem ocorrendo hoje o que vem sendo chamado de "McDonaldização" do comportamento das crianças. A cultura do 'fast food' domina: a satisfação instantânea, adequada a um estilo de vida aonde tudo é feito com pressa, com resultados imediatos, consumíveis, com pouca preocupação com a qualidade do produto, já que o mais importante é consumir e garantir que os consumidores sejam fidelizados aos produtos que aprenderam a consumir. Hoje temos milhões de crianças dependentes do diagnóstico psiquiátrico e dos 
psicotrópicos prescritos. Os consumidores desses produtos irão se transformar em adolescentes e adultos. Potenciais consumidores da psiquiatria ao longo de toda a vida.

\section{REFERÊNCIAS}

A.D.D. RESOURCE CENTER. Proven, Practical Programs and Services for People Dealing with the Challenges of ADHD and Related Issues. https://www.addrc.org. Acessado em 10.9.2019.

AMERICAN PSYCHIATRIC ASSOCIATION. Diagnostic and Statistical Manual of Mental Disorders (DSM-II), 1962.

AMERICAN PSYCHIATRIC ASSOCIATION. DSM-III, 1980.

AMERICAN PSYCHIATRIC ASSOCIATION. DSM-III-R, 1987.

AMERICAN PSYCHIATRIC ASSOCIATION. DSM-IV, 1994.

AMERICAN PSYCHIATRIC ASSOCIATION. DSM-5, 2013.

BARKLEY, Russel. Transtorno do déficit de atenção/hiperatividade - TDAH: guia completo para pais, professores e profissionais da saúde. Porto Alegre: Artmed, 2002.

BARKLEY, Russel A. Against the Status Quo: Revising the Diagnostic Criteria for ADHD. Journal of the American Academy of Child and Adolescent Psychiatry, 2010, vol. 49 (3): 205-207.

BRUSCHMÜLLER, Katrin; MARGROF, Jürgen; SCHNEIDER, Silvia. Is ADHD diagnosed in accord with diagnostic criteria? Overdiagnosis and influence of client gender in diagnosis. Journal of Consulting and Clinical Psychology, 2012, vol 80 (1): 128-138.

CAREY, William B. Problems in Diagnosing Attention Activity. Pediatrics, March 1999, vol. 103 (3), 664.

CORRIGAN, Michael; WHITAKER, Robert. Lancet Psychiatry needs to retract the ADHD study. Enigma Study. www.madinamerica.com , April, 15, 2017.

https://www.madinamerica.com/2017/04/lancet-psychiatry-needs-to-retract-the-adhd-enigmastudy/ Acessado em 22 de setembro de 2019.

CORRIGAN, Michael. The 7 minute ADHD diagnosis.

https://www.youtube.com/watch?v=Gj5VYYMeUZk\&feature=youtu.be Acessado em $18 \mathrm{de}$ Outubro de 2019.

DÍAS, Alícia; JIMÉNEZ, Juan; RODRÍGUEZ, Cristina; AFONSO, Miguel; ARTILES, Ceferino. Consideraciones de los estúdios de prevalência del trastorno por déficit de atención con o sin hiperactividad (TDAH). Revista de Psicologia y Educación, 8(2), 155-170, 2013. 
EPSTEIN, Jeffery; LOREN, Richard. Changes in the Definition of ADHD in DSM-5: Subtle but Important. Neuropsychiatry (London). 2013 Oct 1; 3(5): 455-458.

ESTADÃO. No Brasil, $\mathbf{4 , 4 \%}$ têm déficit de atenção.

https://www.estadao.com.br/noticias/geral,no-brasil-4-4-tem-deficit-de-atencao-imp-,718134 Acessado em 10.9.2019.

FARAÓNE, Stephen ; SERGEANT, Joseph Antony; BIEDERMAN, Joseph. The worldwide prevalence of ADHD: is it an American condition? World Psychiatry: Official Journal of the World Psychiatric Association (WPA), 2(2), 104-113, 2003.

FREITAS, Fernando; AMARANTE, Paulo. Medicalização em Psiquiatria. Rio de Janeiro: Editora FIOCRUZ, 2a . edição, 2017.

HODGKINS, Paul; DOSH, Jalpa; KAHLE, Jennifer; SIKIRICA, Vanja; CANGELOSI, Michael; SETYAWAY, Juliana; ERDER, Moshe; NEWMAN, Peter. A systematic review of global publication trends regarding long-term outeomes of ADHD. Frontiers in Psychiatry, 2(JAN), 1-18, 2012.

HOOGMAN, Martine: BRAALTEN, Janita; HIBAR, Derrek; MENNES, Maarten, et. Al. Subcortical brain volume differences in participants with attention deficit hyperactivity disorder in children and adults: a cross-sectional mega-analysis. Lancet Psychiatry, vol. 4, issue 4, April 01, 2017.

SECRETARIA MUNICIPAL DE EDUCAÇÃO DE LUZIÂNIA/GO. Artigo Material de Apoio para o Professor trabalhar com alunos com Transtorno de Déficit de Atenção e Hiperatividade -TDAH, Secretaria Municipal de Educação de Luziânia/GO, Divisão de Atendimento a Necessidades Educacionais Especiais, 2013.

SILVA, Ana Beatriz Barbosa. Mentes Inquietas - TDAH - Desatenção, Hiperatividade e Impulsividade, Rio de Janeiro: Objetiva, 2009.

STILL, George. Some abnormal psychical conditions in children - Lecture I. The Lancet, 1008-1012, 1902.

\section{SOBRE OS AUTORES:}

\section{Michael W. Corrigan}

PHD em Psicologia. Professor titular da Marshall University e professor titular da Faculdade de Educação e Ecologia Humana da Ohio State University. Autor do livro Debunking ADHS: 10 Reasons to Stop Drugging Kids for Acting Like Kids, pela Ed. Rowman \& Littlefield, 2014. E-mail: corrigan@marshall.edu

\section{iD http://orcid.org/0000-0001-9900-1174}




\section{Robert Whitaker}

Jornalista Investigativo. Autor de livros como: Anatomia de uma Epidemia, RJ: Editora Fiocruz, 2017; com Lisa Cosgrove, Psychiatry under Influence, Palgrave Macmillan, 2015. É o fundador e editor-chefe do site www.madinamerica.com

E-mail: rwhitaker@madinamerica.com

(iD) http://orcid.org/0000-0002-4690-1950

\section{Fernando Freitas}

Doutor em Psicologia (Université Catholique de Louvain - Bélgique).

Pesquisador titular do Laboratório de Estudos e Pesquisas em Psiquiatria Social e Atenção Psicossocial (LAPS/ENSP/FIOCRUZ). Coautor, com Paulo Amarante, do livro Medicalização em Psiquiatria, $2^{\mathrm{a}}$. edição revista, 2015. E coeditor, com Paulo Amarante, do site www.madinbrasil.org

E-mail: ffreitas@ensp.fiocruz.br

iD http://orcid.org/0000-0002-0365-0880

Recebido em: 05 de setembro de 2019 